\title{
Combination Treatment With Proton Pump Inhibitor Plus Prokinetic for Gastroesophageal Reflux Disease
}

TO THE EDITOR: We read with great interest the recent study by Jung et $\mathrm{al}^{1}$ entitled "A systematic review and meta-analysis of randomized control trials: combination treatment with proton pump inhibitor plus prokinetic for gastroesophageal reflux disease." The authors are commended for a well-written paper involving 16 randomized control trials with 1446 participants. The authors concluded that in patients with gastroesophageal reflux disease a combination therapy of prokinetic with proton pump inhibitor is better than proton pump inhibitor alone. While we have great appreciation for the work conducted by the authors there some methodological aspects of this study that we feel need to be pointed out.

Firstly, the authors mentioned that this study was performed in accordance with the Preferred Reporting Items for Systematic reviews and Meta-Analyses (PRISMA) guidelines, however a protocol was not registered in the International Prospective Register of Systematic Reviews (PROSPERO) or any other similar registry such as Cochrane Library and International Platform of Registered Systematic Review and Meta-analysis Protocols (INPLASY). ${ }^{2}$ Registration promotes transparency in the review process, it helps in minimizing the risk of outcome and reporting bias and prevents identical systematic reviews from being performed. ${ }^{3}$

Secondly, the authors mentioned that EMBASE, Cochrane Library, PubMed databases were searched for this study, but they are not enough. For a more comprehensive search the authors should have included other databases such as BIOSIS preview, NLM Gateway, clinicaltrails.gov, and grey literature in their search strategy. Furthermore, the authors failed to provide the search string for this study thus affecting the reproducibility of the search methods.

Thirdly, the authors failed to evaluate the risk of publication bias. Recognition of publication bias is an important step when conducting a meta-analysis, publication bias also referred to as the "filedrawer problem" is often attributed to unpublished or unreported clinical studies which have not been published since they report negative or not significant results leading to a higher proportion of studies with significant results being published. ${ }^{4}$ Publication bias overestimates the potential benefits of an interventions and leads to misrepresentation of adverse effects of a therapeutic modality. Presence of publication bias in systematic review and meta-analysis is problematic as such a bias may invalidate the conclusions reached. ${ }^{5}$ Some commonly utilized tools to assess this bias are funnel plot, Egger's Regression test, and Duval and Tweedie's trim and fill. ${ }^{6,7}$ In the present study an evaluation for publication bias would have significantly improved the credibility of findings.

We hope that the authors will address the concerns raised which will only serve to benefit the research community at large. We once again would like to congratulate the authors on their findings.

\section{Muhammad Ali Tariq* and Bilal Ahmed Department of Internal Medicine, Dow University Hospital, Dow International Medical College, Karachi, Pakistan}

1. Jung DH, Huh CW, Lee SK, Park JC, Shin SK, Lee YC. A Systematic review and meta-analysis of randomized control trials: combination treatment with proton pump inhibitor plus prokinetic for gastroesophageal reflux disease. J Neurogastroenterol Motil 2021;27:165-175.

2. Liberati A, Altman DG, Tetzlaff J, et al. The PRISMA statement for reporting systematic reviews and meta-analyses of studies that evaluate healthcare interventions: explanation and elaboration. BMJ 2009;339:b2700.

3. Moher D, Booth A, Stewart L. How to reduce unnecessary duplication: use PROSPERO. BJOG 2014;121:784-786.

4. Lin L, Chu H. Quantifying publication bias in meta-analysis. Biometrics 2018;74:785-794.

5. Sutton AJ, Song F, Gilbody SM, Abrams KR. Modelling publication bias in meta-analysis: a review. Stat Methods Med Res 2000;9:421-445.

6. Shi L, Lin L. The trim-and-fill method for publication bias: practical guidelines and recommendations based on a large database of metaanalyses. Medicine (Baltimore) 2019;98:e15987.

7. Egger M, Smith GD, Schneider M, Minder C. Bias in meta-analysis detected by a simple, graphical test. BMJ 1997;315:629-634.

Financial support: None.

Conflicts of interest: None.

Author contributions: Muhammad Ali Tariq and Bilal Ahmed equally contributed in writing the manuscript. Both authors approve the final draft of the manuscript. 